\title{
One-Year Clinical Outcome of Inspiron Stent in All-Comers Population (Analysis from 790 Consecutive Patients)
}

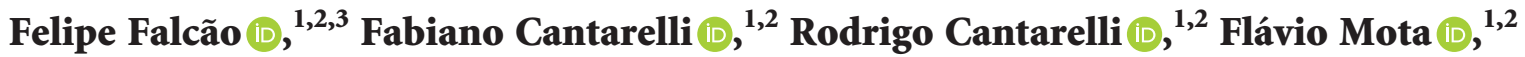 \\ Manuela Navarro $\mathbb{D}^{1},{ }^{1,2}$ Henrique Mota $\mathbb{D}^{1},{ }^{1}$ Martinelly Santos $\mathbb{D}^{1,2}$ Daniel Cruz ${ }^{10},^{2}$ \\ André Sansônio $\left(\mathbb{D},{ }^{1}\right.$ Marcelo Parente $\mathbb{D}^{1},{ }^{1}$ and Flávio Oliveira $\mathbb{I D}^{1,2}$ \\ ${ }^{1}$ Hospital Dom Helder Câmara, Cabo de Santo Agostinho, Brazil \\ ${ }^{2}$ Unidade de Cardiologia Invasiva (UCI)-Hospital Memorial São José, Rede D’Or São Luiz, Recife, Brazil \\ ${ }^{3}$ Department of Internal Medicine, University of Pernambuco, Garanhuns, Brazil \\ Correspondence should be addressed to Felipe Falcão; felipejaf@gmail.com
}

Received 6 June 2020; Revised 30 November 2020; Accepted 8 December 2020; Published 17 December 2020

Academic Editor: Paul M. Grossman

Copyright (C) 2020 Felipe Falcão et al. This is an open access article distributed under the Creative Commons Attribution License, which permits unrestricted use, distribution, and reproduction in any medium, provided the original work is properly cited.

\begin{abstract}
Aims. The goal of this study was to evaluate the performance of the Inspiron ${ }^{\mathrm{TM}}$ coronary stent (Scitech Medical $^{\mathrm{TM}}$, Goiás, Brazil). The Inspiron ${ }^{\mathrm{TM}}$ sirolimus-eluting stent uses an ultrathin L-605 cobalt-chromium alloy with a $75 \mu \mathrm{m}$ strut thickness platform coated with an abluminal biodegradable polymer. The polymer is eliminated from the body through the tricarboxylic acid cycle in 6-9 months, releasing $80 \%$ of the drug within 30 days after its deployment. Methods. It was a prospective, single-center registry. To represent clinical practice, all patients undergoing percutaneous coronary intervention were included in this registry. There were no exclusion criteria. Clinical follow-ups were performed at twelve months. The endpoints were the occurrence of all-cause death, definite stent thrombosis, and new revascularization. Results. Between November 2017 and May 2019, 790 patients were included (1067 lesions). The mean age was $60.42 \pm 14.94$ years, and $74.7 \%$ presented with acute coronary syndrome. Diabetes mellitus was present in $43.9 \%$ of patients, and previous myocardial infarction and previous percutaneous coronary intervention were present in $17.9 \%$ and $11.3 \%$, respectively. Angiographic success was achieved in $99.1 \%$. The incidence of all-cause death was $11.5 \%$ (6.2\% inhospital and $5.3 \%$ in the follow-up) and definitive stent thrombosis was $0.2 \%$. New revascularization was performed in only $5.8 \%$ (target lesion revascularization: $2.2 \%$; progression of disease in another lesion: $3.6 \%$ ). Based on the multivariate regression analysis, only chronic renal failure was an independent predictor of adverse events (OR: 3.3; 95\% CI: 1.22-8.92). Conclusion. The result of this single-center registry demonstrates the safety and excellent performance of the Inspiron ${ }^{\mathrm{TM}}$ stent in daily clinical practice with a low rate of adverse cardiac events.
\end{abstract}

\section{Introduction}

A major reduction in the risk of cardiac events has been achieved with new drug-eluting stents (DES). These stents represented a development of early-generation technology, including polymers with enhanced biocompatibility (permanent or biodegradable), exclusively sirolimus-analogue active drugs, and stents with thin struts $(50-100 \mu \mathrm{m})$ composed of stainless steel, cobalt chromium, or platinum chromium. New-generation DES have higher efficacy and safety in comparison with both early-generation DES and bare metal stents [1].

The Inspiron ${ }^{\mathrm{TM}}$ (Scitech Medical ${ }^{\mathrm{TM}}$, Goiás, Brazil) sirolimus-eluting stent uses an ultrathin L-605 cobalt-chromium alloy with a $75 \mu \mathrm{m}$ strut thickness platform coated with an abluminal biodegradable polymer. The polymer is 
eliminated from the body through the tricarboxylic acid cycle in 6-9 months, releasing $80 \%$ of the drug within 30 days after its deployment [2].

This study aims to evaluate Inspiron $^{\mathrm{TM}}$ in real-world scenarios in challenging conditions.

\section{Methods}

This study was an observational, prospective study at a single center. We included patients undergoing percutaneous coronary intervention (PCI) between November 2017 and May 2019.

The only inclusion criterion was patients with clear indication of PCI (symptoms of angina pectoris and/or documented ischemia or acute coronary syndrome). There were no exclusion criteria. The choice of access route, size of stents, and interventional strategy was entirely left to the judgment of the operator. Inspiron ${ }^{\mathrm{TM}}$ was the only stent available in our center during the study. All procedures were performed according to standard techniques [3, 4]. The local Institutional Committee on Human Research approved the study protocol, and all patients provided written informed consent to participate.

Demographic and clinical data were recorded in a dedicated database for all patients. Procedural success was defined as achieving a minimum stenosis diameter reduction to less than $10 \%$ in the infarct-related artery, along with TIMI (thrombolysis in myocardial infarction) grade flow $\geq 3$ without angiographic complication. TIMI flow was assessed as previously reported [5]. Patients were followed up for one year.

Aspirin was initiated before the procedure and maintained lifelong. A loading dose of clopidogrel was administered for patients not receiving these medications before the procedure. Dual antiplatelet therapy was continued for one year.

The primary endpoint was the occurrence of all-cause death. Secondary endpoints were definitive stent thrombosis (ST) and new revascularization. Definitive ST was defined as angiographic confirmation of thrombus that originates in the stent or in the segment $5 \mathrm{~mm}$ proximal or distal to the stent, with or without vessel occlusion, which is associated with acute onset of ischemic symptoms at rest or electrocardiographic signs of acute ischemia or typical rise and fall of cardiac biomarkers within 48 hours of angiography [6].

Continuous variables were expressed as means and standard deviations or as the median and the 25th and 75th percentiles, according to the presence or absence of a normal distribution, as evaluated by the Kolmogorov-Smirnov test. The Mann-Whitney test and Student's $t$-test were used for continuous variables in accordance by the distribution. Categorical variables were expressed as absolute numbers and percentages. SPSS software version 20 (IBM, Armonk, NY, USA) was used for all analyses, and a final $p$ value less than 0.05 was considered significant.

Univariate and multivariate regression analyses were used to identify potential independent predictors of adverse events. The model was performed using forward stepwise selection. Odds ratios (OR) and their respective confidence intervals $(95 \% \mathrm{CI})$ were used to quantify the effects.

\section{Results}

Seven hundred ninety patients were treated in our center during the study with 1376 Inspiron $^{\mathrm{TM}}$. The number of lesions treated was 1067.

Overall, the mean age was $60.42( \pm 14.94)$ years, $57 \%$ were male, and $74.7 \%$ presented with acute coronary syndrome (ACS). Diabetes mellitus was present in $43.9 \%$ of patients, and previous myocardial infarction and previous PCI were in $17.9 \%$ and $11.3 \%$, respectively (Table 1 ).

Procedural characteristics are presented in Table 2. Left anterior descending PCI was performed in $47.1 \%$. Directing stenting was possible in $33.9 \%$ of the lesions, and the number of stents used per lesion was $1.29 \pm 0.57$. Angiographic success was achieved in $99.1 \%$.

Up to 12 months, clinical follow-up was available in 741 patients $(93.7 \%)$. The incidence of all-cause death was $11.5 \%$ (6.2\% in-hospital and 5.3\% in the follow-up) and definitive ST was $0.2 \%$. New revascularization was performed in only $5.8 \%$ (target lesion revascularization: $2.2 \%$; progression of disease in another lesion: 3.6\%) (Table 3). Based on the multivariate regression analysis, only chronic renal failure was an independent predictor of adverse events (OR: 3.3; 95\% CI: 1.22-8.92).

\section{Discussion}

In this real-world study, without exclusion criteria, this sirolimus stent showed safety and excellent performance in daily clinical practice with a low rate of adverse cardiac events. To the best of our knowledge, this is the first study that enrolled patients with STEMI (primary PCI) for Inspiron $^{\mathrm{TM}}$ DES.

INSPIRON-I trial was a first-in-man evaluation of the safety and efficacy of the Inspiron ${ }^{\mathrm{TM}}$. It was a multicenter, randomized trial, to compare the Inspiron ${ }^{\mathrm{TM}}$ with a bare metal stent with the same metallic structure (Cronus ${ }^{\mathrm{TM}}$ ). At six months, the in-segment late lumen loss was reduced in the Inspiron ${ }^{\mathrm{TM}}$ group $(0.19 \pm 0.16 \mathrm{~mm}$ vs. $0.58 \pm 0.4 \mathrm{~mm}$, respectively; $p<0.001)$, as well as the percent neointimal obstruction $(7.8 \pm 7.1 \%$ vs. $26.5 \pm 11.4 \%$; $p<0.001)$. At four years of follow-up, there was no early or late stent thrombosis, despite the fact that INSPIRON-I trial was not powered for clinical events (only 60 lesions enrolled) [7, 8].

In the DESTINY trial, 170 patients were randomized in the ratio $2: 1$ for Inspiron $^{\mathrm{TM}}$ or Biomatrix ${ }^{\mathrm{TM}}$ (biolimuseluting stent), respectively. The primary endpoint of in-stent late lumen loss was not significantly different between the stents. The relatively limited sample size did not permit a formal comparison between the study groups for the risk of clinical adverse events [9].

An intravascular imaging substudy from the DESTINY trial evaluated 70 patients with intravascular ultrasound (IVUS) and 25 patients with optical coherence tomography (OCT) after 9 months. The main endpoints were percent of neointimal tissue obstruction by IVUS and neointimal strut 
TABLE 1: Baseline clinical characteristics of patients with the Inspiron ${ }^{\mathrm{TM}}$.

\begin{tabular}{lr}
\hline Variables & Inspiron $^{\text {TM }}(n=790)$ \\
\hline Male & $451(57.1 \%)$ \\
Age & $60.42 \pm 14.94$ \\
Hypertension & $700(89.9 \%)$ \\
Diabetes mellitus & $347(43.9 \%)$ \\
Current smoker & $178(34.2)$ \\
Family history of CAD & $45(7.4 \%)$ \\
Chronic renal failure & $39(5.1 \%)$ \\
Previous MI & $137(17.9 \%)$ \\
Heart failure & $19(2.5 \%)$ \\
Previous PCI & $84(11.3 \%)$ \\
Previous CABG & $23(3 \%)$ \\
\hline Clinical presentation & \\
$\quad$ Stable angina & $200(25.3 \%)$ \\
$\quad$ Non-STEMI & $271(34.3 \%)$ \\
$\quad$ STEMI & $319(40.4 \%)$ \\
\hline
\end{tabular}

Values are expressed as $n$ (\%) or mean \pm SD. CAD: coronary artery disease; MI: myocardial infarction; PCI: percutaneous coronary intervention; CABG: coronary artery bypass graft; STEMI: ST elevation myocardial infarction.

TABle 2: Angiographic characteristics.

\begin{tabular}{lc}
\hline & Lesions $(n=1067)$ \\
\hline Target vessel & $277(26 \%)$ \\
RCA & $503(47.1 \%)$ \\
LAD & $252(23.6 \%)$ \\
LCX & $24(2.2 \%)$ \\
LM & $11(1.1 \%)$ \\
Bypass graft & 1.35 \\
\hline Number of lesions treated/patient & \\
\hline TIMI flow before procedure & $366(34.3 \%)$ \\
$<2$ & $701(65.7 \%)$ \\
$\geq 2$ & $49(4.6 \%)$ \\
Restenotic lesions & $133(12.5 \%)$ \\
Bifurcation involved & $705(66.1 \%)$ \\
Predilatation & $1.29 \pm 0.57$ \\
Numbers of stents used per lesion & $29.4 \pm 16.1$ \\
Total stent length per lesion & $1058(99.1 \%)$ \\
Angiographic success &
\end{tabular}

Values are expressed as $n$ (\%). LAD: left anterior descending coronary artery; RCA: right coronary artery; LCX: left circumflex artery; LM: left main; TIMI: thrombolysis in myocardial infarction flow.

TABLE 3: Major adverse cardiac events in twelve months.

\begin{tabular}{lc}
\hline Variables & Inspiron $^{\mathrm{TM}}(n=741)$ \\
\hline All-cause death & $85(11.4 \%)$ \\
Definite ST & $15(0.2 \%)$ \\
\hline New revascularization & $43(5.8 \%)$ \\
Target lesion revascularization & $16(2.1 \%)$ \\
Progression of disease in another lesion & $26(3.5 \%)$ \\
\hline
\end{tabular}

ST: stent thrombosis.

coverage by OCT. Patients treated with both DES had very little neointimal hyperplasia either by IVUS (percent of neointimal hyperplasia obstruction of $4.9 \pm 4.1 \%$ with Inspiron vs. $2.7 \pm 2.9 \%$ with Biomatrix; $p=0.03$ ) or by OCT (neointimal thickness of $144.2 \pm 72.5 \mu \mathrm{m}$ Inspiron $^{\mathrm{TM}}$ vs. $115.0 \pm 53.9 \mu \mathrm{m}$ with Biomatrix $\left.{ }^{\mathrm{TM}} ; p=0.45\right)$. Regarding
OCT strut-level assessment, again both devices showed excellent performance, with high rates of strut coverage $\left(99.49 \pm 1.01 \%\right.$ with Inspiron $^{\mathrm{TM}}$ vs. $97.62 \pm 2.21 \%$ with Biomatrix $\left.^{\mathrm{TM}} ; p<0.001\right)$ and very rare malapposition $\left(0.29 \pm 1.06 \%\right.$ with Inspiron $^{\mathrm{TM}}$ vs. $0.53 \pm 0.82 \%$ with Biomatrix $\left.^{\mathrm{TM}} ; p=0.44\right)$. Patients with any uncovered struts were more frequently identified in the Biomatrix ${ }^{\mathrm{TM}}$ group (9.78 \pm 7.13 vs. $2.29 \pm 3.91 \% ; p<0.001)$. This imaging analysis showed that both DES were effective in terms of suppressing excessive neointimal response, with very high rates of apposed and covered struts, suggesting a consistent and benign healing pattern [10].

Prado Jr. et al demonstrated low rates of events in 470 patients treated with Inspiron ${ }^{\mathrm{TM}}$. At 300 days of follow-up, ST and all-cause death were 0.4 and $2.8 \%$, respectively. Stable coronary disease was the reason for PCI in almost $60 \%$ of patients. Also, few patients were treated after a recent acute myocardial infarction, and none received stents in the context of primary PCI [2].

In comparison with other DES, Inspiron ${ }^{\mathrm{TM}}$ presented rates of definitive ST and TLR similar to historical cohorts previously published (Table 4). It is important to highlight that our population was basically constituted by ACS patients when compared to other registries. The risk of adverse events is distinct to patients with stable disease, mainly in relation to mortality [11-15].

In our trial, Inspiron ${ }^{\mathrm{TM}}$ was used as a workhorse stent in a center specialized in ACS. Although our all-cause death was high, it is comparable to other studies in similar situations [16-18]. A reason that could be responsible for this rate is the real-world scenario associated with ACS. The socalled off-label indications are the majority in our center. Heavy calcification, older patients, multivessel disease, and low ejection fraction are rules and not exceptions.

Some limitations must be considered. First, it was a registry in a center for ACS treatment, so extrapolation of this data should be done to patients with similar characteristics. Multicenter randomized controlled trials should be done to confirm our findings. Second, the analysis of 
TABLE 4: Comparison between Inspiron and other DES (1-year follow-up).

\begin{tabular}{lccccc}
\hline & Inspiron $^{\mathrm{TM}}{ }^{\text {current }}$ registry & Promus $^{\mathrm{TM}, 12}$ & Synergy $^{\mathrm{TM}, 13}$ & Resolute Onyx $^{\mathrm{TM}, 11}$ & Ultimaster $^{\mathrm{TM}, 14}$ \\
\hline Number of patients & 790 & 988 & 846 & 252 & 1660 \\
Diabetes & $43.9 \%$ & $35.2 \%$ & $31.1 \%$ & $46.2 \%$ & $29 \%$ \\
Non-STEMI & $34.3 \%$ & $7.3 \%$ & $25.9 \%$ & $24.4 \%$ & $12.5 \%$ \\
STEMI & $40.4 \%$ & $5.8 \%$ & 0 & $0.8 \%$ & $10.5 \%$ \\
Stent thrombosis & $0.2 \%$ & $0.5 \%$ & $0.4 \%$ & $0.7 \%$ & $0.9 \%$ \\
Target lesion revascularization & $2.1 \%$ & $1.9 \%$ & $2.0 \%$ & $4.4 \%$ & $3.2 \%$ \\
\hline
\end{tabular}

outcomes was limited to twelve months. So, this study is not able to make any conclusions regarding very long-term prognosis.

\section{Conclusion}

In this single-center, real-world experience, Inspiron ${ }^{\mathrm{TM}}$ stent proved to be safe and demonstrated excellent performance.

\section{Data Availability}

The datasets generated during the current study are available in the https://figshare.com/ repository, named as Corehemodata.

\section{Conflicts of Interest}

The authors declare no conflicts of interest.

\section{References}

[1] F. J. Neumann, M. Sousa-Uva, A. Ahlsson et al., "2018 ESC/ EACTS guidelines on myocardial revascularization," EuroIntervention, vol. 14, pp. 1435-1534, 2019.

[2] G. F. Prado, E. E. Ribeiro, P. H. Melo et al., "Clinical performance of a novel ultrathin strut, low-dose, sirolimuseluting stent with abluminal-only biodegradable polymeric coating for patients undergoing percutaneous coronary intervention in the daily practice," Cardiovascular Diagnosis and Therapy, vol. 5, no. 6, pp. 414-419, 2015.

[3] B. Ibanez, S. James, S. Agewall et al., "2017 ESC guidelines for the management of acute myocardial infarction in patients presenting with ST-segment elevation: the task force for the management of acute myocardial infarction in patients presenting with ST-segment elevation of the European Society of Cardiology (ESC)," European Heart Journal, vol. 39, pp. 119-177, 2018.

[4] G. N. Levine, E. R. Bates, J. C. Blankenship et al., "2011 ACCF/ AHA/SCAI guideline for percutaneous coronary intervention," Journal of the American College of Cardiology, vol. 58, no. 24, pp. e44-e122, 2011.

[5] TIMI Study Group, "The thrombolysis in myocardial infarction (TIMI) trial: phase I findings," New England Journal of Medicine, vol. 312, pp. 932-936, 1985.

[6] R. Mehran, S. V. Rao, D. L. Bhatt et al., "Standardized bleeding definitions for cardiovascular clinical trials," Circulation, vol. 123, no. 23, pp. 2736-2747, 2011.

[7] E. E. Ribeiro, C. M. Campos, H. B. Ribeiro et al., "First-in-man randomised comparison of a novel sirolimus-eluting stent with abluminal biodegradable polymer and thin-strut cobaltchromium alloy: INSPIRON-I trial," EuroIntervention, vol. 9, no. 12, pp. 1380-1384, 2014.
[8] M. D. Oliveira, E. E. Ribeiro, C. M. Campos et al., "Four-year clinical follow-up of the first-in-man randomized comparison of a novel sirolimus eluting stent with abluminal biodegradable polymer and ultra-thin strut cobalt-chromium alloy: the INSPIRON-I trial," Cardiovascular Diagnosis and Therapy, vol. 5, no. 4, pp. 264-270, 2015.

[9] P. A. Lemos, A. A. C. Abizaid, G. C. Meireles et al., "Metallic limus-eluting stents abluminally coated with biodegradable polymers: angiographic and clinical comparison of a novel ultra-thin sirolimus stent versus biolimus stent in the DESTINY randomized trial," Cardiovascular Therapeutics, vol. 33, no. 6, pp. 367-371, 2015.

[10] J. R. Costa, J. Daniel, C. Alexandre et al., "Intravascular imaging comparison of two metallic limus-eluting stents abluminally coated with biodegradable polymers: IVUS and OCT results of the DESTINY trial," International Journal of Cardiovascular Imaging, vol. 33, pp. 1-8, 2016.

[11] C. C. Tam, K. Chan, S. Lam et al., "One-year clinical outcomes of patients implanted with a Resolute Onyx zotarolimuseluting stent," Journal of International Medical Research, vol. 46, no. 1, pp. 457-463, 2018.

[12] C. F. de Souza, J. Ribamar Costa, A. Abizaid et al., "POLAR registry (promus eluting stent registry in Latin America): 1 year follow-up results," Revista Brasileira de Cardiologia Invasiva (English Edition), vol. 20, no. 1, pp. 29-34, 2012.

[13] D. J. Kereiakes, I. T. Meredith, S. Windecker et al., "Efficacy and safety of a novel bioabsorbable polymer-coated, everolimus-eluting coronary stent," Circulation Cardiovascular Interventions, vol. 8, pp. 1-8, 2015.

[14] A. Beneduce, G. Ferrante, A. Ielasi et al., "One-year clinical outcome of biodegradable polymer sirolimus-eluting stent in diabetic patients: insight from the ULISSE registry (ULtimaster Italian multicenter all comerS Stent rEgistry)," Catheterization and Cardiovascular Interventions, vol. 260, pp. 36-41, 2020.

[15] T. Pilgrim, P. Vranckx, M. Valgimigli et al., "Risk and timing of recurrent ischemic events among patients with stable ischemic heart disease, non-ST-segment elevation acute coronary syndrome, and ST-segment elevation myocardial infarction," American Heart Journal, vol. 175, pp. 56-65, 2016.

[16] L. S. Piegas, A. Avezum, H. P. Guimarães et al., "Acute coronary syndrome behavior: results of a brazilian registry," Arquivos brasileiros de cardiologia, vol. 100, no. 6, pp. 502-510, 2013.

[17] A. C. Caluza, A. H. Brbosa, I. Gonçalves et al., "ST-elevation myocardial infarction network: systematization in 205 cases reduced clinical events in the public health care system," Arquivous Brasileiros de Cardiologia, vol. 99, no. 5, pp. 991040-991048, 2012.

[18] L. A. 1. P. e. Mattos, O. Berwanger, E. S. d. Santos et al., "Desfechos clínicos aos 30 dias do registro brasileiro das síndromes coronárias agudas (ACCEPT)," Arquivos Brasileiros de Cardiologia, vol. 100, no. 1, pp. 6-13, 2013. 